\title{
Are bitewings of value in epidemiological studies of dental caries?
}

\author{
Does the use of bitewing radiographs provide additional benefit over clinical \\ examination alone when assessing caries prevalence in populations of young \\ people?
}

\begin{abstract}
Bloemendal E, de Vet HC, Bouter LM. The value of bitewing radiographs in epidemiological caries research: a systematic review of the literature. J Dent 2004; 32:255-264
\end{abstract}

Data sources Sources used were Medline, reference lists of relevant papers and additional papers suggested by Dutch experts in the field. Study selection Only papers written in English or Dutch and published after 1990 were included. Studies were selected only if they were conducted in patients $<24$ years of age and they included the use of bitewings and clinical examination. Studies of extracted teeth were excluded. Studies were assessed for quality.

Data extraction and synthesis A methodological quality-assessment checklist was completed to assess internal and external validity for each study. A two-by-two table of radiographic versus clinical data was constructed for each study. These data were used to assess figures for radiographic and clinical prevalence and to find a conversion factor to calculate the total prevalence from the clinical prevalence.

Results Seven studies met the inclusion criteria. In the approximal surfaces the radiographic prevalence was considerably higher than the clinical prevalence. The conversion factors required to calculate the total prevalence from the clinical prevalence varied considerably across studies. In the occlusal surfaces the radiographic and clinical prevalence was similar, but extra lesions were detected with both methods. The seven studies included in this review show that there is variation in the figures for radiographic and clinical prevalence of caries, depending on the type of surfaces examined. On the approximal surfaces figures for radiographic prevalence are substantially higher than those for clinical prevalence, but on the occlusal surfaces the radiographic and clinical prevalence is approximately the same for the caries into dentine.

Conclusions To study trends in the prevalence of caries, radiographic examinations are not necessary, but to assess the prevalence of caries they do have additional but unknown value.

\begin{abstract}
Commentary
Epidemiological surveys to assess point-prevalence and trends in dental caries are conducted in numerous countries world-wide, as can be seen from the World Health Organization Oral Health Country/Area Profile Programme (see www. whocollab.od.mah.se/ index.html). The use of radiographs in epidemiological surveys of dental health is not widespread, however, because most surveys are conducted for the purpose of assessing and monitoring disease prevalence and not for treatment planning. There is of course an ethical issue of exposure to radiation to take radiographs, which may not be of significant benefit to the exposed individuals. In addition it is not clear what additional yield, over a clinical examination, the use of radiographs would offer in the survey setting.
\end{abstract}

This review article set out to determine exactly what extra yield was afforded by the addition of bitewing radiographs and found seven studies that met the inclusion criteria. All but one study, which used the enamel caries threshold, reported that bitewing radiographs detected more lesions on approximal surfaces (1.7$10.0 \%$ extra dentinal lesions and $5.4-13.6 \%$ extra enamel lesions) than were detected clinically $(0.03-0.40 \%$ extra dentinal lesions and $0.1-8.7 \%$ extra enamel lesions) but there was little difference on occlusal surfaces. The review found that the use of bitewing radiographs added little value to the process of monitoring trends in dental caries.

It should be noted, however, that there was no gold standard in any of these studies to determine whether or not the lesions detected were true carious lesions. The accuracy of bitewing radiographs for reliable detection of caries has been investigated in numerous studies and the reported sensitivity and specificity for caries detection varies considerably. This will also vary with the threshold of detection selected and the experience of the practitioner making the diagnosis, as well as the examination conditions, eg, use of magnification and masking from unwanted light sources.

It is difficult to draw conclusions from this review as there are inherent differences in the populations that may affect the diagnostic yield when using bitewing radiographs, such as the age of those sampled and the severity of carious lesions. For example, the Machiulskiene study, ${ }^{1}$ which is cited in this review, sampled 11- and 12-year old children, in whom many approximal sites on the posterior teeth might be expected to directly visible, thus affecting the additional value of the radiograph. The review does usefully draw attention to the value of further work that might produce stratified conversion factors to apply to prevalence data from clinical examination; this would help to estimate true prevalence without the need for bitewing radiographs.

\section{Practice point}

- There is insufficient evidence at present to indicate the additional diagnostic yield from using bitewing radiographs in an epidemiological study of specific populations.

\section{Gail Topping}

Dental Caries Control Programme Director, Dental Health Services Research Unit, University of Dundee, Dundee, Scotland, $U K$

1. Machiuliskiene V, Nyvad B, Baelum V. A comparison of clinical and radiographic caries diagnoses in posterior teeth of 12 year old Lithuanian children. Caries Res 1999; 33:340-348.

Evidence-Based Dentistry (2004) 5, 72. doi:10.1038/sj.ebd.6400264 